\title{
X-raying the Hubble Frontier Fields cluster Abell 2744
}

\section{Dominique Eckert}

Department of Astronomy, University of Geneva, Ch. dEcogia 16, 1290 Versoix, Switzerland email: Dominique.Eckert@unige.ch

\begin{abstract}
Abell 2744 was the first HFF cluster completed. It displays a fascinating complexity in its distribution of dark and luminous matter, which led to its nickname of the Pandora cluster. In late 2014 we obtained a deep $(110 \mathrm{ks})$ observation of this cluster with XMM-Newton, with the aim of making a detailed comparison between the optical, X-ray and lensing properties of this system. The new X-ray observation unveiled the presence of three hot gas filaments extending on scales of several Mpc and connected to the cluster core. The X-ray structures coincide spatially with the distribution of galaxies and dark matter and provide strong evidence for the existence of the elusive warm-hot intergalactic medium (WHIM). The new observation also reveals the complexity of the thermodynamic structure of the cluster core and a probable shock front associated with the radio relic located $1 \mathrm{Mpc} \mathrm{NW}$ of the cluster core.
\end{abstract}

Keywords. galaxies: clusters: individual (Abell 2744), X-rays: galaxies: clusters, gravitational lensing, large-scale structure of universe

\section{Introduction}

Galaxy clusters are the largest virialized structures in the present Universe. They are located at the nodes of the cosmic web and form through the continuous merging and accretion of smaller structures (Springel et al. (2006)). In the present epoch, this phenomenon is observable in the outer regions of massive clusters, where infalling galaxies and galaxy groups are located. On the larger scales, galaxy clusters are connected to the filaments of the cosmic web. These filaments might retain a large fraction of the Universe's baryons (Cen \& Ostriker (1999), Davé et al. (2001)), which should be in the form of a low-density gas in the temperature range $10^{5}-10^{7} \mathrm{~K}$ known as the warm-hot intergalactic medium (WHIM). For all these reasons, studying the distribution of warm and luminous matter in the faint outskirts of galaxy clusters is essential for our understanding of the processes leading to the formation of virialized structures in the Universe.

Abell 2744 ( $z=0.306$, Boschin et al.(2006), Owers et al. (2011)) was the first cluster for which the HFF observations were completed. This very massive cluster $(M(<1.3 \mathrm{Mpc})=$ $1.8 \times 10^{15} M_{\odot}$, Merten et al. (2011)) exhibits a complex distribution of dark and luminous matter in its central region. From pre-HFF HST and Subaru/SubprimeCam data, Merten et al. (2011) presented a detailed weak-lensing map of the cluster core and identified the presence of at least 4 cluster-scale halos in the central $1 \mathrm{Mpc}$. On the other hand, X-ray observations only reveal two prominent gas concentrations (Kempner \& David (2004), Owers et al. (2011)), including a main region of X-ray emission encompassing all 4 clumps detected in the weak-lensing analysis, and a mysterious second clump located North-West of the main mass concentration with no apparent lensing counterpart. The amazing complexity of this system lead Merten et al. (2011) to suggest the nickname of Pandora cluster to highlight the wealth of information to be collected through the study of this remarkable cluster. 

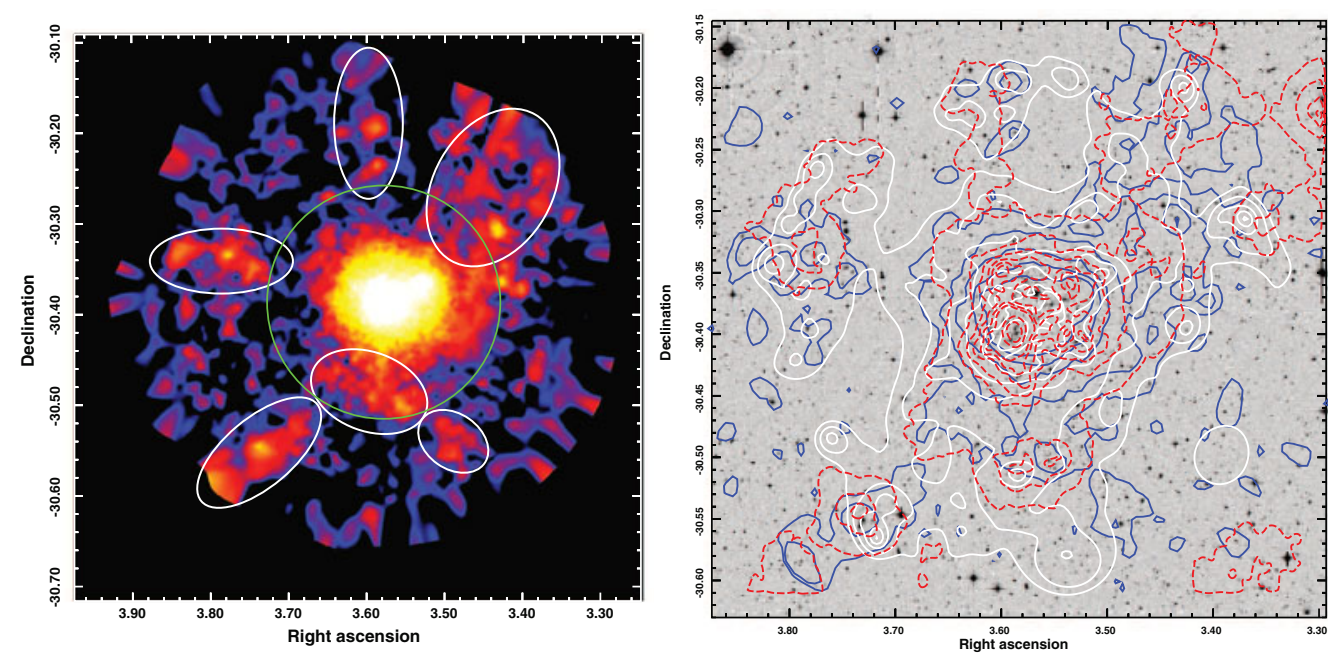

Figure 1. From Eckert et al. (2015). Left: Adaptively-smoothed XMM-Newton image of the hot gas in Abell 2744 in the [0.5-1.2] keV band. The circle shows the approximate location of the cluster's virial radius. The white ellipses highlight regions of significant soft X-ray emission. Right: CFHT/Megacam i'-band image of the cluster and its surroundings. The contours show the X-ray emission (dark), optical light (dashed), and mass map reconstructed through weak lensing (white).

While the core of Abell 2744 has already been studied in detail, the outer regions of this system (beyond $1 \mathrm{Mpc}$ from the cluster core) are still unexplored. In the framework of the multiwavelength campaign accompanying the HFF initiative, we proposed a deep observation of this cluster with XMM-Newton (PI: Kneib) to map the large-scale structure surrounding Abell 2744 and study the thermodynamic properties of the gas in its central regions. This observation was performed in December 2014 (OBSID 074385) and revealed a wealth of previously unknown features. Here we summarize some of the findings obtained thanks to the new XMM-Newton data.

\section{Hot gas filling the cosmic web}

We reduced the XMM-Newton data using the XMMSASv13.5 software package and created a map of the cluster and its surroundings in the [0.5-1.2] keV band, where the sensitivity of the telescope is the highest. We then applied an adaptive smoothing algorithm to enhance faint features. The resulting image is shown in the left-hand panel of Fig. 1 (from Eckert et al. (2015)).

Beyond the cluster's virial radius (approximated by the green circle in Fig. 1) we observed several regions of significant diffuse emission $(\mathrm{S} / \mathrm{N}>6 \sigma)$ radially connected to Abell 2744. Unlike the cluster's core, these features are not visible at higher energies $(>2 \mathrm{keV})$, which indicates that the gas filling these structures is cooler than the intracluster medium (ICM) of Abell 2744. To identify these structures, we used a large set of spectroscopic redshifts made available by Owers et al. (2011) and selected the galaxies whose receding velocity falls within $\pm 5,000 \mathrm{~km} / \mathrm{s}$ from the cluster mean. We found that the structures observed in the NW, S/SW, and E of the cluster coincide with filaments of galaxies at the cluster's redshift. Therefore, the structures detected in X-rays are likely associated with hot gas filling the cosmic web over projected scales of $\geqslant 8 \mathrm{Mpc}$. 

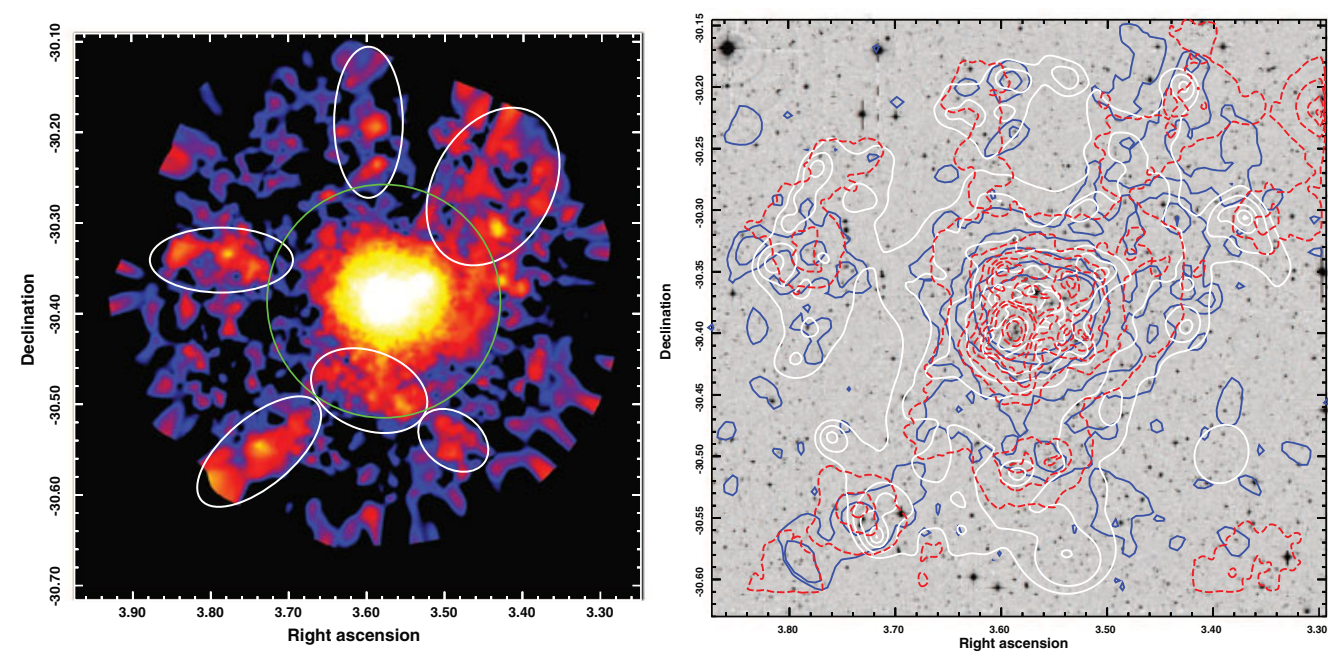

Figure 2. Properties of the gas around the radio relic. Left: X-ray surface brightness profile of the gas in a sector encompassing the radio relic. The position of the relic is shown by the arrow. Right: Temperature map of the gas in the region. The contours show the radio emission at 610 $\mathrm{GHz}$ from the GMRT (image courtesy H. Intema). The gas with the highest temperature $(>14$ $\mathrm{keV}$ ) is found around the location of the radio relic.

In addition, we used archival CFHT and WFI/ESO-2.2m observations of the cluster in the BVRi bands to select background galaxies and cluster members in order to calculate the weak-lensing signal using the non-parametric method developed by Jullo \& Kneib (2009). The resulting mass contours are shown in the right-hand panel of Fig. 1 on top of the CFHT image and compared to the X-ray emission and light distribution. An excess lensing signal was observed in the direction of the filaments, which proves the association of the X-ray structures with the cosmic web.

From a spectral analysis of the X-ray signal in the filaments, we determined that the gas in these structures has a temperature of about $1 \mathrm{keV}\left(\sim 10^{7} \mathrm{~K}\right)$ and a baryonic over-density of $\sim 200$. These values are very similar to the expectations for the hottest and densest phase of the WHIM Davé et al. (2001). The gas fraction of the filaments is $5-15 \%$. Therefore, our observations provide strong indications that a significant fraction of the Universe's baryons are filling the cosmic web in a warm-hot phase, as suggested by numerical simulations.

\section{Gas heating and particle acceleration at cosmological shock waves}

Major cluster mergers induced by structure-formation processes are the most energetic events since the big bang, releasing energies as large as $10^{64}$ erg (e.g. Ricker \& Sarazin (2001)). The collision between the gaseous atmospheres of the merging subclusters induces Mpc-scale outgoing shock waves with Mach numbers in the range 2-4 (Vazza et al. (2010)). These shock waves accelerate particles to relativistic energies, giving rise to the phenomenon known as radio relics, i.e. diffuse, irregular radio structures in the periphery of galaxy clusters (e.g. Bonafede et al. (2012)). In addition, cosmological shock waves inject entropy in the gas of the merging structures, raising the temperature of the ICM. The link between shock waves, particle acceleration and ICM thermodynamical properties is still poorly understood (e.g. Ogrean et al. (2013), Vazza et al. (2015)). 
Abell 2744 is known to host a spectacular example of radio relic located $\sim 1 \mathrm{Mpc} \mathrm{NE}$ of the cluster core (Orrú et al. (2007)). The depth of our new XMM-Newton observation of the cluster allowed us to reach a high signal-to-noise ratio in the relic area. Thus, we extracted a temperature map of the ICM in this region by using an adaptive binning technique and fitting a spectral template to pseudo-spectra obtained from the count rates measured in 5 logarithmically-spaced energy bands. In the right-hand panel of Fig. 2 we show the corresponding temperature map with radio contours from GMRT at $330 \mathrm{GHz}$ overlaid (courtesy H. Intema). This analysis reveals the presence of very hot $(T>14$ $\mathrm{keV}$ ) gas coinciding with the position of the radio relic. In the left-hand panel of Fig. 2 we show the surface-brightness profile of the X-ray emission in the region of the radio relic. We detected a clear break in the surface-brightness profile at the position of the relic, which indicates the presence of a discontinuity. This feature is likely a shock front travelling outwards from the core in the NE direction. The gas in the downstream region has been heated up by the moving shock wave.

\section{Conclusions}

It has been known for some time that the Pandora cluster Abell 2744 is an exceptionally complex system. Thanks to its wide field of view and high sensitivity, XMM-Newton is the best X-ray telescope currently available to look at the big picture and understand the properties of the large-scale environment of massive clusters. Our new observation has revealed the presence of warm-hot gas associated with the accretion flow from the cosmic web (Eckert et al. (2015), see Fig. 1), which allow us to study the properties of the WHIM in unprecedented details. This deep observation also allowed us to characterize the properties of the intracluster gas in the region surrounding the radio relic, revealing the presence of a shock front associated with the radio edge and of shock-heated gas in the downstream region (see Fig. 2). The detailed comparison between our high-quality thermodynamic maps and the extremely deep mass maps obtained during the HFF program will undoubtedly lead to exciting new advances in our understanding of cluster formation processes.

\section{References}

Bonafede, A., Brüggen, M., van Weeren, R., et al. 2012, MNRAS, 426, 40

Boschin, W., Girardi, M., Spolaor, M., \& Barrena, R. 2006, A\&\&A, 449, 461

Cen, R. \& Ostriker, J. P. 1999, ApJ, 514, 1

Davé, R., Cen, R., Ostriker, J. P., et al. 2001, ApJ, 552, 473

Eckert, D., Jauzac, M., Shan, H. Y. et al. 2015, Nature, in press

Jullo, E. \& Kneib, J.-P. 2009, MNRAS, 395, 1319

Merten, J., Coe, D., Dupke, R., et al. 2011, MNRAS, 417, 333

Ogrean, G. A., Brüggen, M., van Weeren, R. J., et al. 2013, MNRAS, 433, 812

Orrú, E., Murgia, M., Feretti, L., et al. 2007, A\& A, 467, 943

Owers, M. S., Randall, S. W., Nulsen, P. E. J., et al. 2011, ApJ, 728, 27

Kempner, J. C. \& David, L. P. 2004, MNRAS, 349, 385

Ricker, P. M. \& Sarazin, C. L. 2001, ApJ, 561, 621

Springel, V., Frenk, C. S., \& White, S. D. M. 2006, Nature, 440, 1137

Vazza, F., Brunetti, G., Gheller, C., \& Brunino, R. 2010, New Astronomy, 15, 695

Vazza, F., Eckert, D., Brüggen, M., \& Huber, B. 2015, MNRAS, 451, 2198 